\title{
Primary and Behavioral Healthcare Integration: Threat or Opportunity for Addiction Treatment Organizations?
}

\section{Linda Rosenberg, MSW}

Nationwide, health care systems are experiencing an increasingly rapid pace of health reforms, especially in burgeoning areas such as health homes, accountable care organizations, which emphasize the growing need for bidirectional integration of primary care and behavioral health. Several articles in this issue of the Journal of Behavioral Health Services \& Research explore care that requires collaboration between primary and behavioral health: "Predictors of primary care physicians' self-reported intention to conduct suicide risk assessments" by Lisa M Hooper et al., "Outcomes associated with a cognitive-behavioral chronic pain management program implemented in three public HIV primary care clinics" by Jodie Anne Trafton et al., and the "Relationship between neighborhood characteristics and recruitment into adolescent family-based substance use prevention programs" by Hilary F. Byrnes et al. However, is this emphasis on integration a threat or opportunity for specialty addiction treatment organizations?

A recent U.S. Substance Abuse and Mental Health Services Administration-U.S. Health Resources and Services Administration Center for Integrated Health Solutions survey ${ }^{1}$ identified a growing number of specialty addictions treatment organizations that partner with community health centers, methadone treatment centers that qualify as Federally Qualified Health Centers, and residential treatment programs that offer primary care services onsite. Even state alcohol and drug authorities are focusing on integration at annual provider meetings and state training academies.

In communities, specialty addiction treatment organizations have long held relationships with primary care practices. Historically, these relationships have ensured physicals for individuals entering residential care, interim services for pregnant women and individuals using intravenous drugs, and, more recently, medication interventions as a part of addiction treatment.

New integration models serve to enhance providers' opportunities to reach individuals in earlier stages of addiction, and the earlier treatment that follows could assuage damage to their bodies and improve overall treatment outcomes. Developing recovery plans which incorporate overall health, including exercise, prevention, and specific public health goals for chronic health problems, support recovery.

In terms of Screening, Brief Intervention, and Referral to Treatment (SBIRT), specialty addiction professionals fill a gap in primary care settings by supporting "referral to treatment." Without this involvement of addiction professionals, SBIRT programs often fail when a patient requires a

Address correspondence to Linda Rosenberg, MSW, National Council for Community Behavioral Healthcare, Rockville, MA, USA. Email: MeenaD@thenationalcouncil.org.

Journal of Behavioral Health Services \& Research, 2012. (C) 2012 National Council for Community Behavioral Healthcare. DOI 10.1007/s11414-012-9278-y 
referral to specialty care, as primary care professionals may not know the available community resources, how to access central diagnostic and referral units, and/or how to determine the level of care needed by a patient. Onsite addiction professionals facilitate referrals by helping determine appropriate levels of care, by helping the patient understand what to expect from specialty care, and by supporting the patient throughout the referral process. Onsite addiction professionals can also provide brief intervention services in the primary care office.

Highly skilled in motivational interviewing, the specialty addiction system brings needed expertise to the primary care setting. Intermittent doses of motivational interviewing and encouragement to attend self-help groups can incite a path to recovery, and recovery coaches can help sustain recovery. Since SBIRT services are often reimbursable in primary care, addiction professionals gain access to new financial resources.

Embedding addiction services in primary care also reduces discrimination and stereotypes. When a patient is introduced to recovery and treatment in the privacy of a primary care doctor's office, it improves engagement in care, provides an opportunity for earlier interventions, and gives the patient a safe place to secure recovery support.

Integrating primary care and behavioral health care is indeed an opportunity, and an expanding model of care in communities. For people living with addictions, only specialty addictions professionals' expertise will optimize integration models. This expertise is key to improving their opportunity for recovery and better overall health, while ensuring cost savings for the overall health care systems.

\section{References}

1. Center for Integrated Health Solutions. Environmental Scan of Integration Efforts within Substance Abuse Specialty Programs. Washington, DC: U.S. Substance Abuse and Mental Health Services Administration and U.S. Health Resources and Services Administration, 2011. 